\title{
«Où va l'humanité?» - L'homme néoténique et/ou le surhomme
}

\section{Jean Martin}

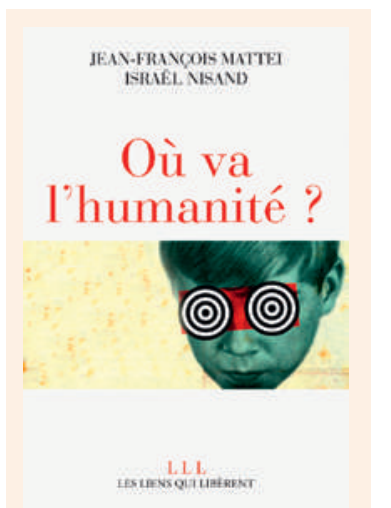

Jean-François Mattei, Israël Nisand Où va l'humanité?

Paris: Editions Les Liens qui Libèrent; 2013.

88 pages. $9,50 €$

ISBN 979-10-209-0060-9

\footnotetext{
* La néoténie, en biologie du
développement, est le
phénomène qui consiste en la

La néoténie, en biologie du
développement, est le
phénomène qui consiste en la

La néoténie, en biologie du
développement, est le
phénomène qui consiste en la conservation de traits juvéniles chez un être adulte. Au cours du XX $\mathrm{X}^{\mathrm{e}}$ siècle, différents auteurs ont formulé différents auteurs ont formul
l'hypothèse que l'homme peut lui aussi être qualifié de néoténique. Cette hypothèse a une dimension anthropologique et philosophique, soulignée par le philosophe Dany-Robert Dufour: Dany-Robert Dufour: l'homme serait dépendant de la relation à l'Autre, d'où la substitution nécessaire, par l'espèce humaine, de la Culture à la Nature. L'homme se réappropriant le monde par la parole, la croyance symbolique et la création de prothèses, c'est-à-dire la technique (D.-R. Dufour, «Il était une fois le dernier homme», Denoël, Paris 2012). Pour Freud, relève aussi Nisand, la néoténie crée le besoin d'amour.
}

Ce petit ouvrage présente des conférences données à Strasbourg en janvier 2013, au troisième Forum européen de bioéthique. Jean François Mattei a été professeur de pédiatrie et génétique, puis député et ministre de la santé; il a joué un rôle-clé dans l'élaboration des lois françaises de bioéthique. Israël Nisand est professeur de gynéco-obstétrique à Strasbourg, où il enseigne aussi les sciences humaines. Tous deux sont des acteurs sociétaux d'importance.

\section{L'homme est un néotène - \\ Biologie et anthropologie}

Dans sa contribution, Nisand discute largement le caractère néoténique de l'être humain. * "Avorton de singe, si immature à la naissance, l'homme n'aurait pas dû survivre tant il est bâclé... Terriblement prématuré, ce qui s'explique aisément car sinon la taille du cerveau aurait été incompatible avec celle du bassin de la mère.»

Point fondamental: «Ces insuffisances ont imposé l'avènement de la culture, qui est venue remplacer les instincts.» Passage de la première nature (celle de l'animal) à la seconde: celle que l'homme «a dû développer pour survivre et qui reprend en les inversant les données de la première nature: «Plus faible je suis, plus fort je dois être»».

Comme cela s'est-il fait? «La fantastique intelligence de l'animal se déploie et s'épuise dans l'instant**. L'homme, lui, pratique assidûment l'aprèscoup. Cette capacité de revenir sur son passé afin d'en tirer des enseignements pour l'avenir le caractérise. Il est aussi le seul à savoir qu'il va mourir [...]. L'instrument de tout ce jeu avec le temps c'est le langage, qui permet de ramener les choses absentes dans le présent. Le langage a sauvé l'homme en permettant la transmission d'un nombre infini de représentations.» Avec son corollaire, l'écriture.

Un homme est donc le «produit de deux logiques: son équipement naturel de base, qui n'a pas changé depuis cent mille ans, et le tissu narratif, artistique et grammatical constituant son environnement de seconde nature». Pratiquement: «La liberté de la main combinée au langage permet la création de prothèses de toutes sortes. Les objets utilisés par le néotène entrent dans un processus cumulatif. La création prothétique vise à modifier matériellement certaines limites qui nous étaient imposées par la nature.»

\section{Améliorer/augmenter l'homme?} Potentialités et possibles dérives

La grande préoccupation des auteurs, c'est le devenir de l'Homme devant les avancées de la science et des techniques. Nisand: «L'homme sait désormais lire l'écriture [génétique] dont il est l'expression et c'est la première fois qu'une créature va pouvoir faire retour dans la création pour se refaire et se poser comme son propre créateur.» Serions-nous à l'orée d'un changement dans la sélection naturelle? Est-il possible qu'une sélection artificielle, voulue et manipulée, soit un jour commandée par les hommes euxmêmes qui jusque-là n'étaient que les objets passifs de cette sélection? [...] Un fantasme d'immortalité justifie à lui seul toutes les recherches qui n'ont plus de chimériques que le nom.»

Pourquoi, demande Nisand sans y croire, devraiton s'arrêter dans les efforts d'amélioration de l'être humain, pourquoi ne chercherait-on pas la post-, trans- ou surhumanité? «Pourquoi l'espèce devraitelle encore compter sur une évolution biologique spontanée si lente et aléatoire?» «Commencer le grand œuvre du câblage entre le vivant et le prothétique [et] inventer une hypernature pour vivre plus longtemps dans une puissance sans frein»?

Il le refuse et met en garde à l'endroit de ce qu'il appelle les grands avides; en bref la course aux profits des individus et des entreprises au mépris du bien commun. "Les néotènes sont en train de devenir un peuple d'uniques où chacun se met en avant et ignore l'autre, en oubliant qu'il a besoin de lui pour être. La convergence de l'hyper-puissance financière avec les considérables moyens techno-scientifiques pourrait faire éclater notre communauté humaine.» Et conduire à l'épuisement et la destruction de la planète. Avec, grand risque certainement, la montée toujours plus accentuée des inégalités: «Les grands avides pourront être tentés de sortir de leur état de néotènes en abandonnant à son sort le reste de l'humanité.» Comme le dit Donna Dickenson à propos de développements qui ne seraient ouverts qu'à un petit nombre d'élus: «But what if we wound up in the unenhanced Lumpenproletariat?»

\section{A propos d'assistance médicale à la procréation}

Nisand par contre n'est guère préoccupé par des évolutions qui inquiètent d'autres en matière de procréation assistée (son champ professionnel - une certaine subjectivité n'est pas exclue). L'aide médicale dans ce domaine ne modifie pas les corps, dit-il. Le diagnostic préimplantatoire est souhaitable pour éviter la transmission d'une maladie héréditaire; les déclarations sur les risques anthropologiques d'accepter la filiation homosexuelle sont sans fondement; l'émoi provoqué par la mise en œuvre de maternité 
** Cette formule est élégante mais rend-elle justice à la réalité? J'y pensai en lisant le dernier ouvrage de Jean-Claude Ameisen, médecin et chercheur, président du Conseil consultatif national français d'éthique («Sur les épaules de Darwin» - «Je t'offrirai des spectacles admirables» Ed. Les Liens qui Libèrent/ France Inter, Paris 2013). Décrivant les connaissances que l'observation de la nature et la recherche ont acquises sur les comportements des animaux sociaux (fourmis, abeilles), ainsi que des oiseaux. A l'évidence des facultés d'apprentissage et de mémorisation extraordinaires - et le mot est faible. Des phénomènes dont on ne peut pas dire qu'ils se déploient seulement dans l'instant. Du registre de l'instinct pour l'essentiel, on ne saurait prétendre qu'il y a conscience. Mais, en général et de diverses façons, la nature (la «création») est faite de continua plutôt que de césures forcément infranchissables; pas de sauts qui font qu'un processus est totalement et intrinsèquement différent de l'autre. Les travaux récents sur les grands primates (chimpanzés, bonobos) montrent des couduites dont il est bien difficile de dire qu'elles n'ont rien à voir avec ce que nous humains faisons sur un mode plus sophistiqué.

*** Dans un éditorial, B. Kiefer [2] évoque l'ouvrage qui vient de sortir de deux pontifes de l'économie numérique américaine (E. Schmidt et J. Cohen, "A nous d'écrire l'avenir», Paris: Denoël, 2014). Parmi ses commentaires: «Sommes-nous nous-mêmes réductibles à nos circuits neuronaux définis par nos organes désormais remplaçables, ou y a-t-il quelque chose d'autre derrière ces circuits? Cela a-t-il un sens de dire Jes? Et plus loin: «Ce qu'il y a d'individuel et de singulier en l'homme ne représenterait, selon le storytelling de Schmidt et Cohen, qu'une sorte d'épiphénomène historique qui s'apprête à disparaître dans les systèmes informatisés, dans le virtuel et la connexion.» de substitution semble disproportionné (je l'ai entendu y donner son accord)

«Modifier le récit de l'origine ne constitue pas pour l'humanité un grand carrefour. Toucher au génome, en revanche, introduire de nouveaux caractères transmissibles, là est la véritable ligne jaune». A discuter.

\section{Le corps, philosophiquement, juridiquement, pratiquement}

Jean-François Mattei, dans son texte «Le corps sera-til encore humain?», discute ce qu'est pour nous le corps, en général et dans la culture française. Rappelant les principes de non-disponibilité et de nonpatrimonialité du corps et de ses parties, particulièrement impératifs en droit français - alors que les doctrines anglo-saxonnes sont plus larges: qu'on pense à l'acceptation de la grossesse pour autrui, à la vente d'ovules par des jeunes femmes volontaires, à des positions favorables à un marché ouvert et explicite d'organes (y compris de donneurs vivants) voire au clonage reproductif.

Dignité de l'être humain: ici aussi, la doctrine française est spécialement ferme; cas échéant, la personne n'a pas la liberté de déterminer librement ce que dignité de son existence veut dire, parce que cette notion a un sens découlant du cadre constitutionnel et légal et qui s'impose à tous, y compris à qui aurait une autre vision. «Au-delà du souci de préserver l'humanité de l'ensemble des hommes, c'est l'humanité de chacun qui pourrait aujourd'hui se trouver menacée, comme si on oubliait que chaque homme possède en lui une parcelle d'une seule et même humanité» (certains philosophes anglosaxons ne se rallieraient pas à cette préoccupation communautaire, seul l'individu importe... There is no such thing as society, a dit une Première Ministre britannique).

«La question se pose avec une acuité grandissante de savoir si, modifiant notre corps, nous pourrions néanmoins continuer d'être humains. Le corps est-il une simple enveloppe dont on pourrait changer ou une partie indissociable de notre humanité?» Personne ne peut se prétendre pur esprit. Et Mattei de citer Michel Foucault: «Ce n'est que par et à travers mon corps que je puis être moi-même». Ce qui rappelle que, plutôt que «'ai un corps», il convient de dire «Je suis un corps», ou «JE est mon corps.»***

\section{Prothèses et transplantations - La question du statut du corps entre personne et chose se pose désormais différemment}

Devrait-on repenser la distinction du droit romain entre choses et personnes? «La science ne montre-telle pas que le corps obéit à des lois semblables à celles qui régissent l'univers des choses?» Ainsi en rapport avec les développements que des prothèses permettent. Celles de type mécanique, comme en orthopédie, ne donnent guère lieu à états d'âme compliqués, mais il peut en aller autrement pour celles du registre électronique et robotique, en direction des cyborgs (combinaisons homme-machine les plus courants étant les personnes avec pacemakers... mais vient maintenant le cœur artificiel). Mattei aborde la problématique de plus en plus présente du enhancement (amélioration/rehaussement de l'être humain), y compris dopage. Il y a ceux qui refusent ces améliorations artificielles et ceux qui pensent que c'est une véritable obligation morale de les rechercher tous azimuts...

S'agissant des transplantations d'organes, on connait les ressentis pleins de questions, ambivalents, parfois perturbants, de personnes greffées. Ils sont liés d'une part à la relation avec le donneur, décédé ou vivant, au pourquoi de son choix de donner, auxrisques que le donneur vivant assume, et d'autre part à la problématique de la vie avec l'organe de quelqu'un d'autre: peu de problèmes du côté du rein, mais il est troublant de vivre avec le cœur (siège traditionnel des émotions, rappelle Mattei) qui a auparavant battu pour/dans un(e) autre. Difficultés possibles avec la greffe de la main - main qui est le prolongement de l'esprit qui l'anime: "Comment peut-on caresser avec la main d'un autre?» Quant à la greffe du visage (je m'étais essayé à y réfléchir - [1]), elle a suscité des réactions «entre émerveillement et stupeur: comment peut-on sourire avec le visage d'un autre? Comment embrasser avec la bouche d'un autre?»

Faut-il croire «les techno-prophètes [des mages douteux] affirmant que les nouvelles technologies vont permettre à l'homme, en s'affranchissant des servitudes corporelles [et de notre finitude?], de retrouver le bonheur du paradis perdu [...] et l'entrée triomphale dans la posthumanité grâce à l'intelligence artificielle?» Ou faut-il craindre que l'homme soit «une espèce en voie de disparition, victime de son propre pouvoir technologique»? (Mattei).

Beaucoup de questions et peu de réponses, mais c'est le lot de beaucoup de ce que à quoi l'éthique (bio- et sociale) réfléchit aujourd'hui. «Il convient d'aimer notre faiblesse, qui nous contraint à la connaissance, et éviter le clivage de l'humanité entre un nouvel homme et les vieux néotènes.» «Nous sommes tous collectivement embarqués dans un train à grande vitesse mais dépourvu de signal d'alarme et de frein», dit Nisand. Mattei et lui apportent à cet égard, sous une forme agréable à lire, des éclairages stimulants.

\section{Références}

1 Martin J. Transplantation du visage - Quels effets psychologiques et sociaux et quels enjeux éthiques et juridiques? Rev Méd Suisse. 2005;1:583-4.

2 Kiefer B. Le bonheur virtuel. Rev Méd Suisse. 2014;10:144. 